\title{
FAILURE OF RANDOM NETWORKS
}

WANSOO T. RHEE

\author{
The Ohio State University \\ 301 Hagerty Hall \\ 1775 College Road \\ Columbus, Ohio 43210
}

(Received March 28, 1988 and in revised form August 22, 1988)

\begin{abstract}
This paper studies random failure of networks when we know the probability of failure of each link but we know nothing about the intercorrelation of these failures. Without any assumption on the structure of the network, we compute the probability that there still exists a path from the source to the sink after the worst possible failure of the network.
\end{abstract}

KEY WORDS: Random graph

1980 AMS SUBJECT CLASSIFICATION CODE. 68C25.

\section{INTRODUCTION.}

A network $N=[V ; A]$ consists of a finite collection $V$ of elements $v, w, \ldots$, together with a subset $A$ of the pairs $(v, w)$ of elements of $V$. The elements of $V$ are called nodes and the elements of $A$ are called links. Each link of the network will be assumed to have an orientation.

Let $s$, the source, and $t$, the sink, be two distinguished nodes of $V$. A path from $s$ to $t$ is a sequence $s=v_{0}, v_{1}, \ldots, v_{k}=t$ with $\left(v_{\imath}, v_{i+1}\right) \in A$ for $0 \leq i<k$. A subnetwork $N^{\prime}$ of $N$ is a network $N^{\prime}=\left[I^{\prime} ; A^{\prime}\right]$ with $A^{\prime} \subseteq A$. We denote $S N$ the set of subnetworks of $N$.

In this paper, we study random failures of $N=[V ; A]$. A random failure of $N$ is an event that removes some of the elements (links) in $A$. Thus the random failure of $N$ produces a (random) subnetwork $N^{\prime}$ of $N$. An each subnetwork has some probability to be the outcome of the random failure. Thus, mathematically, we can model a random failure of $N$ by a probability measure $P$ on $S N$.

The problem studied in this paper has been suggested by P. Erdös and A. Hajnal [1]. We suppose we know the probability $p_{(v, w)}$ of failure of each link $(v, w) \in A$, 


$$
p_{(v, w)}=P\left(\left\{N^{\prime} \in S N: N^{\prime}=\left[V ; A^{\prime}\right],(v, w) \notin A^{\prime}, A^{\prime} \subseteq A\right\}\right),
$$

but otherwise we know nothing about the probability $P$ on $S N$. We address the following question: when can we conclude that with positive probability there is a path from $s$ to $t$ ?

\section{MAIN RESULTS.}

We define the following quantity of a network $N=[V ; A]$. This quantity measures the worst case of failure for the network.

DEFINITION. The failure-resistant factor of $N$ given $\left\{p_{(v, w)}:(v, w)\right.$ is a link of $\left.N\right\}$ is $\inf \left\{P\left(N^{\prime} \in S N\right.\right.$ : there exists a path in $N^{\prime}$ from $s$ to $\left.\left.t\right)\right\}$

where the infimum is taken over all probabilities $P$ on $S N$ satisfying condition (1).

The following theorem computes the failure-resistant factor of a network. It is a remarkable fact that the result holds for any type of network configuration.

THEOREM. For a network $N=[V ; A]$, the failure-resistant factor is equal to $1-a$ where $a=1 \wedge \inf \left\{\sum_{0 \leq 1<k} p_{\left(v_{1}, v_{1+1}\right)}\right\}$, the infimum being taken over the set of all paths $s=v_{0}, v_{1}, \ldots, v_{k}=t$ from $s$ to $t$.

The main appeal of our result lies in its generality. On the other hand, saying nothing about the intercorrelation of the failures of different links allows e.g. negative correlation between these failures, which is not a typical behaviour. It would be of interest to have general estimates of the failure-resistant factor of a network for other notions of failure resistance, where the infimum in the definition of failure resistance is not taken over all probabilities of random failures satisfying (1), but on a smaller set, e.g. by adding the restriction that the correlation between the failures of different links has to be positive.

PROOF OF THEOREM. Consider a path $s=v_{0}, v_{1}, \ldots, v_{k}=t$ from $s$ to $t$ such that $\sum_{0 \leq i<k} p_{\left(v_{t}, v_{t+1}\right)}<1$. For any random failure $P$ satisfying (1),

$$
\begin{gathered}
P\left(\left\{N^{\prime} \in S N ; N^{\prime}=\left[V ; A^{\prime}\right],\left(v_{i}, v_{i+1}\right) \in A^{\prime} \text { for each } 0 \leq i<k\right\}\right) \\
\geq 1-\sum_{0 \leq i<k} p_{\left(v_{i}, v_{i+1}\right)} .
\end{gathered}
$$

Since this inequality is also true for all the paths from $s$ to $t$, we get $r \geq 1-a$ where $r$ is the failure-resistant factor.

The difficulty is to prove that $r \leq 1-a$, so we have to construct a suitable probability on $S N$. For each $s$ in $V$, define $g(z)=\inf \left(\sum_{0 \leq_{i<k}} p_{\left(v_{t}, v_{1+1}\right)}\right)$, where the infimum is taken over all the paths $s=v_{0}, v_{1}, \ldots, v_{k}=z$ (we set $\left.g(s)=0\right)$. We set $f(z)=1 \wedge g(z)$, so $a=f(t)$. For each pair $(v, w)$ in $A$, we have 


$$
f(w) \leq f(v)+p_{(v, w)}, \quad f(v) \leq f(w)+p_{(v, w)}
$$

so $|f(v)-f(w)| \leq p_{(v, w)}$. So we can find an interval $F_{(v, w)} \subset[0,1]$ of length $p_{(v, w)}$ that contains both $f(v)$ and $f(w)$. We now define the map $h$ fror: $[0,1]$ to $S N$ as follows. For $\alpha$ in $[0,1]$,

$$
h(\alpha)=\left[V ;\left\{(v, w) \in A: \alpha \notin F_{(v, w)}\right\}\right] .
$$

We define the probability $P$ on $S N$ by writing, $P\left(\left\{N^{\prime}\right\}\right)=\lambda\left(h^{-1}\left(N^{\prime}\right)\right)$ for each $N^{\prime}$ in $S N$, where $\lambda$ is lebesgue measure. By definition of $h$, we observe that $(v, w) \notin h(\alpha)$ if and only $\alpha \in F_{(v, w)}$, and, since $\lambda\left(F_{(v, w)}\right)=p_{(v, w)}$ this implies that $P$ satisfies (1). We have to show that if

$$
W=\left\{N^{\prime} \in S N ; \text { there exists a path in } N^{\prime} \text { from } s \text { to } t\right\},
$$

we have $P(W) \leq 1-a$. It is enough to prove that if $\alpha<a, h(\alpha) \notin W$, because then

$$
P(W)=\lambda\left(h^{-1}(W)\right) \leq \lambda([a, 1])=1-a .
$$

We fix $\alpha<a$. Suppose, if possible that there is a path $s=v_{0}, v_{1}, \ldots, v_{k}=t$ in $h(\alpha)$ from $s$ to $t$. Then we have $f(s) \leq \alpha<f\left(v_{k}\right)=a$, so there is a largest $\ell<k$ for which $f\left(v_{\ell}\right) \leq \alpha$. We then have $f\left(v_{\ell+1}\right)>\alpha$, so $f\left(v_{\ell}\right) \leq \alpha<f\left(v_{\ell+1}\right)$. The definition of $F_{\left(v_{\ell}, v_{\ell+1}\right)}$ shows that $\alpha \in F_{\left(v_{\ell}, v_{\ell+1}\right)}$. The definition of $h$ shows that $\left(v_{\ell}, v_{\ell+1}\right) \notin h(\alpha)$. This contradiction concludes the proof.

\section{REFERENCES}

1. P. Erdös and A. Hajnal, Some remarks on set theory IX, Combinatorial problems in measure theory and set theory, Michigan Math. J. 11, (1964) 107-127. 


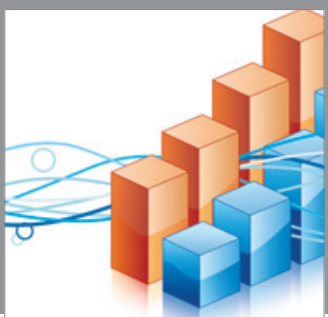

Advances in

Operations Research

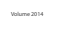

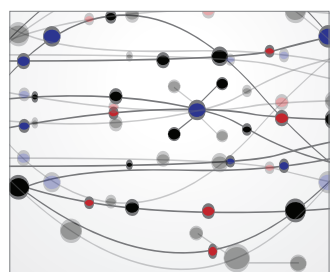

\section{The Scientific} World Journal
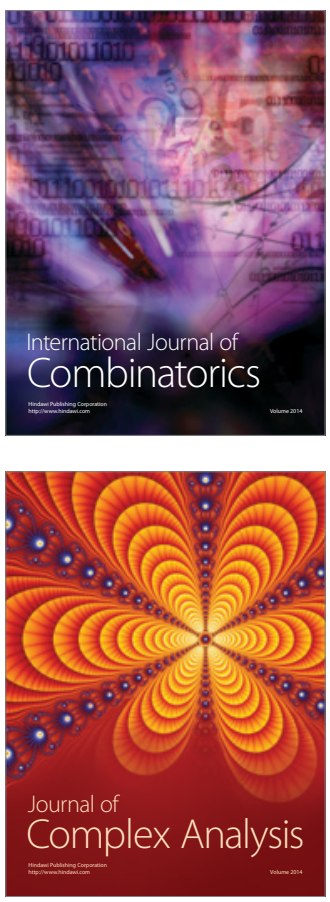

International Journal of

Mathematics and

Mathematical

Sciences
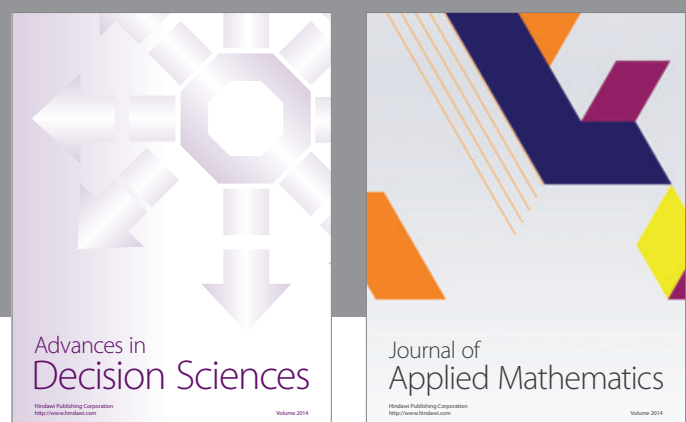

Journal of

Applied Mathematics
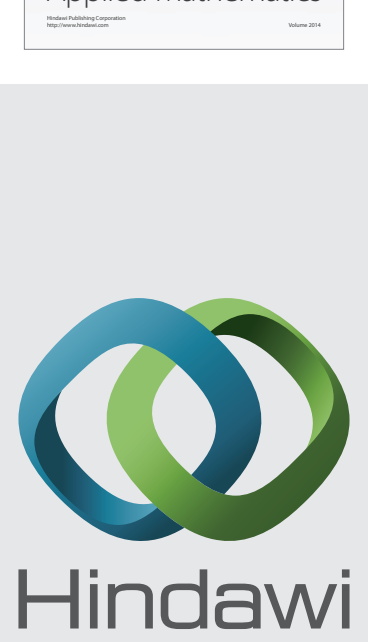

Submit your manuscripts at http://www.hindawi.com
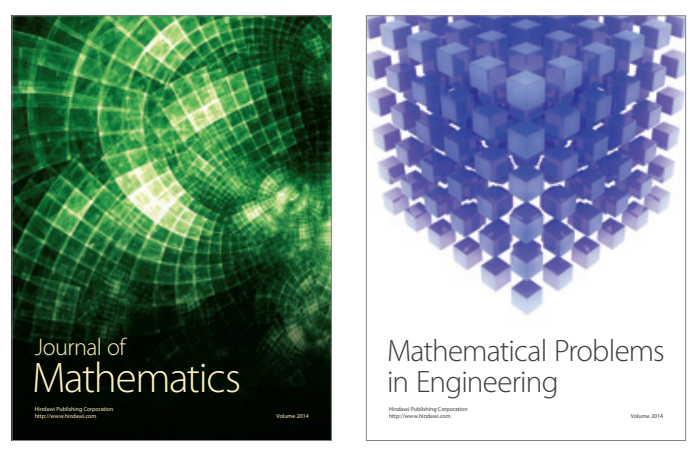

Mathematical Problems in Engineering
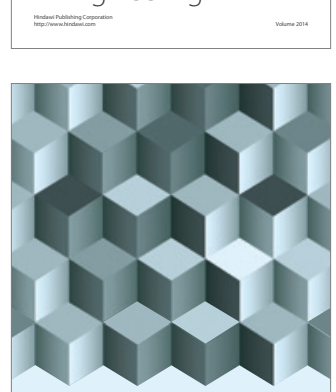

Journal of

Function Spaces
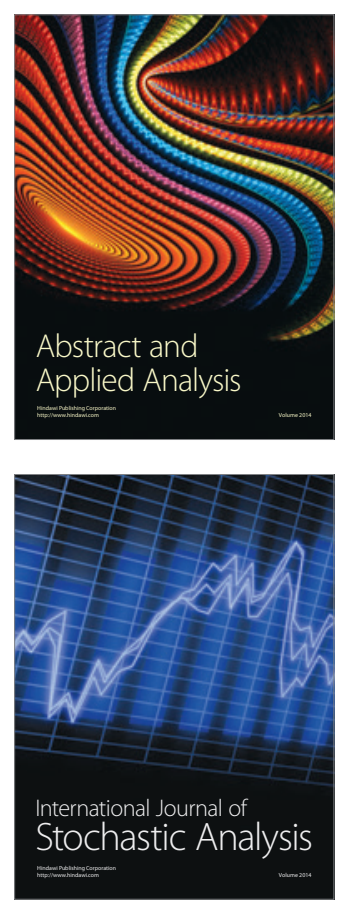

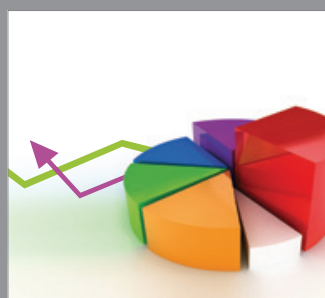

ournal of

Probability and Statistics

Promensencen
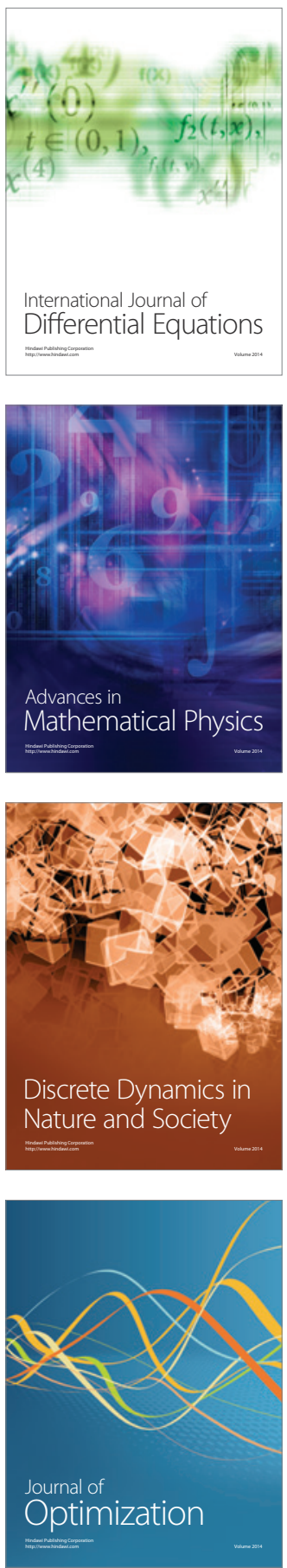